\title{
IMPACT PARAMETER DESCRIPTION OF MULTIPARTICLE AND ELASTIC SCATTERING
}

\author{
F.S. HENYEY \\ Randall Laboratory of Physics, University of Michigan, Ann Arbor, Michigan 48104, USA
}

Received 6 June 1973

\begin{abstract}
Impact parameter variables are defined for a multiparticle production process. The equation of unitarity for elastic scattering is written at high energy in terms of these variables. The overall impact parameter can be expressed in terms of the impact parameters of all the produced particles. The unitarity equation becomes an "optical theorem" at each impact parameter - diffractive scattering is given by beam depletion. These features allow this technique to give a much clearer interpretation of unitarity in any model than has therefore been possible. This technique can be used to study existing models, and to suggest new ones.
\end{abstract}

Diffractive scattering at high energy is generally thought to be a rather direct consequence of unitarity. If a rèasonably correct description of the bulk of high energy processes were to exist, one could readily calculate diffraction - that is, the part of high energy scattering described by the "Pomeron". Only for such scattering do the contributions to unitarity dominantly interfere constructively.

However, no sufficiently good description of high energy processes exists. Most of the cross section is due to production processes, which are considerably less well understood than two-body processes. There are several competing models for these multiparticle production processes, none of which is in a sufficiently refined form to realiably calculate elastic (and other diffractive) scattering.

Until now, when a calculation based on any of these models has failed to reproduce the experimental results for elastic scattering (or when the calculation has succeeded in agreeing with some features of the data), it has not been clear whether the calculation should be taken seriously. Perhaps the failure was a consequence of some details or perhaps a consequence of general features of the model. It is the purpose of this work to remedy this defect in our ability to comprehend the structure of unitarity for elastic scattering. We wish to understand which features of the multiparticle amplitude are important in determining elastic scattering.

There are two distinct types of features which might be important. First are those which are apparent in the data - such things as transverse momentum distributions, multiplicities, etc. These are features of the absolute square of the amplitude, since they show up in the cross section. Second are features related to the phase of the amplitude, and how the phase depends on the momenta of the particles. There is no way of deducing this phase directly from any multiparticle data. (In the case of particles with spin, relative phases between different amplitudes can be determined by polarization measurements. The unknown phase is the overall phase, which depends on the momenta of all the produced particles.)

In the case of two-body scattering at high energy, questions of unitarity and phase have profitably been studied by use of impact parameter as the relevant variable. This suggests that we attempt to understand multiparticle processes as well in the framework of an impact parameter description. The impact parameter is the transverse position of a scattering particle, that is, it is its position in a plane perpendicular to the scattering direction. Thus we will introduce a transverse position, or impact parameter, for each of the produced particles. These impact parameters are, then, just the variables conjugate to the transverse momenta.

As a scattering process occurs, all longitudinal positions are occupied at some time. Therefore there is no apparent advantage in replacing longitudinal momenta. We shall use the longitudinal momentum of each particle (or its rapidity) as the longitudinal variable. We shall see that this is a fortunate choice. In contrast, in the high energy

\footnotetext{
Work supported by the U.S. Atomic Energy Commission.
} 
approximation in which the scattering proceeds primarily in one direction (the beam direction), only one transverse position is occupied by each particle. For this reason, impact parameter will be a useful description.

For two-body scattering there is only one impact parameter, not one for each particle. This is a result of the choice of the origin in transverse space. The origin is chosen at one particle and the impact parameter is the position of the other. We shall see that a similar phenomenon occurs for multiparticle states. In this case the arbitrariness of the choice of origin is more apparent. The choice is made to result in a slight calculational convenience; this choice, however, has absolutely no influence on the physics.

So far, our discussion has lacked precision, and has been primarily motivational. For the idea to have real value it must be made precise, with controlled high energy approximations. Thus we next turn to the definition of impact parameter in multiparticle states, and to a calculation of unitarity for elastic scattering.

The impact parameter $\boldsymbol{b}_{j}$ is defined as the variable conjugate to the transverse momentum $\boldsymbol{k}_{j} . \boldsymbol{b}_{j}$ and $\boldsymbol{k}_{j}$ are two dimensional vectors. An $n$-particle final state amplitude is transformed to

$\widetilde{M}\left(\boldsymbol{b}_{j}, p_{j}\right)=\int \prod_{j=1}^{n}\left[\frac{\mathrm{d}^{2} \boldsymbol{k}_{j} \exp \left(\mathrm{i} \boldsymbol{k}_{j} \cdot \boldsymbol{b}_{j}\right)}{(2 \pi)^{2}}\right] M\left(\boldsymbol{k}_{j}, p_{j}\right) \delta^{2}\left(\sum_{j=1}^{n} \boldsymbol{k}_{j}\right)$.

The $p_{j}$ 's are longitudinal momenta, and are not transformed.

The momentum conservation delta function makes this slightly different from an ordinary Fourier transform. $\widetilde{M}$ is, as a result, only a function of differences of $b$ 's. A small amount of algebra establishes the inverse transformation:

$M\left(\boldsymbol{k}_{j}, p_{j}\right)=(2 \pi n)^{2} \int \prod_{j=1}^{n}\left[\mathrm{~d}^{2} \boldsymbol{b}_{j} \exp \left(-\mathrm{i} \boldsymbol{k}_{j} \cdot \boldsymbol{b}_{j}\right)\right] \tilde{M}\left(\boldsymbol{b}_{j}, p_{j}\right) \delta^{2}\left(\sum_{j=1}^{n} \boldsymbol{b}_{j}-\boldsymbol{B}_{\mathrm{o}}\right)$.

$B_{\mathrm{o}}$ is comple tely arbitrary; since (transverse) momentum is conserved, there is an invariance under translations $\boldsymbol{b}_{j} \rightarrow \boldsymbol{b}_{j}+\left(\boldsymbol{B}_{\mathrm{o}}-\boldsymbol{B}_{\mathrm{o}}^{\prime}\right) / \boldsymbol{n}$. In what follows we will choose $\boldsymbol{B}_{\mathrm{o}}=0$ for simplicity. With this choice it is not necessary to make explicit the dependence of the matrix element only on differences on $b$ 's; that is

$b_{j}=b_{j}-\frac{1}{n} \sum_{j^{\prime}} b_{j^{\prime}}$

We wish to compute the imaginary part of elastic scattering at all values of the impact parameter, or equivalently at all momentum transfers for which $t \ll s$. In order to do this, we introduce a set of high-energy approximations, paralleling the usual high energy approximations for two-body impact parameter. We use the rapidity $\left.y_{j}=\frac{1}{2} \ln \left(E_{j}+p_{j}\right) / E_{j}-p_{j}\right)$ as the longitudinal variable. With this choice, if a particle is fast, its energy is almost independent of its transverse momentum, $E_{j} \approx p_{j}$ (fast particle). If, on the other hand, it is slow, it contributes little to the energy and longitudinal momentum conservation conditions (i.e. to del ta functions in the unitarity equation), except in an average sense if there are many slow particles. Thus we assume $\Sigma E_{j}=\sqrt{s}$ and $\Sigma p_{j}=0$ can be approximately written in terms of only the $y_{j}$ 's. Related approximations are involved in the rotation of the multiparticle state by an angle which rotates the initial state of elastic scattering to the final state. This rotation is given by $p_{j} \rightarrow p_{j}-k_{j} \cdot \Delta / p$ and $k_{j} \rightarrow k_{j}+p_{j} \Delta / p$ where $\Delta$ is the momentum transfer in elastic scattering, $t \approx-\Delta^{2}$ and $p$ is the incident center of mass momentum $s \approx 4 p^{2}$, and we have assumed $\Delta^{2} \ll p^{2}$. We make the approximations that the matrix element does not vary under the small shift in $p_{j}$, and that the $p_{j}$ in the shift of $k_{j}$ can be taken independent of variations in $k_{j}$, either because $p_{j}$ is large compared to $k_{j}$ and is approximately independent of $k_{j}$, or it is small and the variations of the term $p_{j} \Delta / p$ as $k_{j}$ is varied are small compared to a typical $k_{j}$. The set of approx. imations we make can be shown to be good by numerical example. (Unless, for some entirely unknown reason, phases vary much more rapidly than magnitudes of amplitudes.)

These approximations allow the transverse kinematics to be separated from the longitudinal kinematics. Unita- ity for the elastic amplitude can be written 


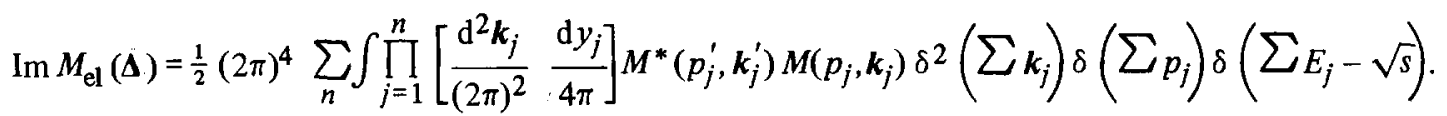

Our approximations allow us to replace $p_{j}^{\prime}$ by $p_{j}$. We then transform $M^{*}$ and $M$ to impact parameters $b_{j}^{\prime}$ and $b_{j}$ respectively.

We have the Fourier exponentials $\exp \left(-\mathrm{i} \boldsymbol{k}_{j} \cdot \boldsymbol{b}_{j}\right)$ and $\exp \left[+\mathrm{i}\left(\boldsymbol{k}_{j}+p_{j} \dot{\boldsymbol{\Delta}} / p\right) \cdot \boldsymbol{b}_{j}^{\prime}\right]$. By virtue of our approxima. tions, the only $k_{j}$ dependence is in these exponentials and in the transverse momentum conservations. Thus we can do the $\boldsymbol{k}_{j}$ integrals explicitly, yielding delta functions of $\boldsymbol{b}_{j}-\boldsymbol{b}_{j}^{\prime}$ ' and we can integrate the $\boldsymbol{b}_{j}^{\prime}$ 's using these delta functions. Thus $M^{*}$ and $M$ are finally evaluated for the same set of coordinates. Carrying out this algebra in detail, with due regard for all the delta functions, we obtain

$$
\begin{aligned}
\operatorname{Im} M_{\mathrm{el}}(\Delta)=\sum_{n} \frac{1}{2}(2 \pi)^{4}(2 \pi n)^{2} \int \prod_{j=1}^{n}\left[\mathrm{~d}^{2} \boldsymbol{b}_{j} \frac{\mathrm{d} y_{j}}{4 \pi}\right] \delta\left(\sum p_{j}\right) \delta\left(\sum E_{j}-\sqrt{s}\right) \\
\quad \times \exp \left(\mathrm{i} \boldsymbol{\Delta} \cdot \sum_{b_{j}} p_{j} / p\right) \delta^{2}\left(\sum_{b_{j}}\right)\left|\tilde{M}\left(\boldsymbol{b}_{j}, p_{j}\right)\right|^{2} .
\end{aligned}
$$

Note again that this only depends on $|\widetilde{M}|^{2}$, rigorously, given our high energy approximations. Finally we transform this to the impact parameter of the elastic scattering

$$
\begin{aligned}
\operatorname{Im} \widetilde{M}_{\mathrm{el}}(\boldsymbol{b})=\sum_{n} \frac{1}{2}(2 \pi)^{4}(2 \pi n)^{2} \int \prod_{j=1}^{n}\left[\mathrm{~d}^{2} \boldsymbol{b}_{j} \frac{\mathrm{d} y_{j}}{4 \pi}\right] \delta\left(\sum p_{j}\right) \delta\left(\sum E_{j}-\sqrt{s}\right) \\
\quad \times \delta^{2}\left(\sum \boldsymbol{b}_{j}\right) \delta^{2}\left(\boldsymbol{b}-\sum_{j=1}^{n} \boldsymbol{b}_{j} p_{j} / p\right)\left|\tilde{M}\left(\boldsymbol{b}_{j}, p_{j}\right)\right|^{2} .
\end{aligned}
$$

This is the basic formula of our approach. (In the case with spin, the amplitudes must be taken to be $s$-channel helicity amplitudes, as they are in the ordinary two particle impact parameter representation.)

The relationship between $M_{\mathrm{el}}(\Delta)$ and $\widetilde{M}_{\mathrm{el}}(b)$ is

$M_{\mathrm{el}}(\boldsymbol{\Delta})=\int \mathrm{d}^{2} \boldsymbol{b} \exp (\mathrm{i} \Delta \cdot \boldsymbol{b}) \widetilde{M}_{\mathrm{el}}(\boldsymbol{b})$,

which differs by a factor $2 \pi$ from the usual Hankel transform.

Eq. (6) can be understood very simply. The longitudinal momentum and energy conservation delta functions are familiar enough. The next delta function (two dimensional) refers to the arbitrariness of the choice of origin in impact parameter space. It could just as well have been $\delta^{2}\left(\Sigma b_{j}-B_{\mathrm{o}}\right)$. It reminds us that $\widetilde{M}$ is really only a function of differences of $b$ 's. The next two-dimensional delta function is new [1]. Many people [e.g. 2], guided by the multiperipheral model and their intuition have assumed either $b=\Sigma B_{j}$ or $\boldsymbol{b}=\boldsymbol{b}_{1}-\boldsymbol{b}_{n}$, where 1 and $n$ refer to, for example, the nucleons in $\mathrm{pp} \rightarrow 2$ nucleons $+(n-2)$ pions. If the first of these is to be an approximation to the correct result, the $B_{j}$ 's must be taken to be differences of our $b_{h}$ 's: $\boldsymbol{B}_{j}=\boldsymbol{b}_{j+1}-\boldsymbol{b}_{j}$. In this case the $\boldsymbol{B}$ 's are the variables conjugate to the transverse part of the multiperipheral momentum transfers: $B_{j} \leftrightarrow Q_{j}, t_{j}=Q_{j}^{2}+$ longitudinal part. With this definition of $\boldsymbol{B}_{j}$, these two common assumptions become equivalent. They represent an approximation to our result. If $p_{1} \approx p, p_{n} \approx-p$, all other $p$ 's $\ll p$, then $\Sigma\left(p_{j} b_{j} / p\right) \approx b_{1}-b_{n}$. However in the absence of a definite leading particle, our formula for $b$ is a much better approximation.

Our expression for $b$ allows a simple interpretation. It can be written as $p b=\Sigma p_{j} b_{j}$. Rotating by $90^{\circ}$, we obtain $\boldsymbol{b} \times \boldsymbol{p}=\Sigma \boldsymbol{b}_{j} \times \boldsymbol{p}_{j}$. Thus the overall impact parameter is given by angular momentum conservation. This is not surprising because the $p_{j} / p$ factor arises from the rotation of the amplitude from the initial to final elastic scattering states. Our approximations have amounted to dropping small complicated parts of the total angular momentum. 
A very important part of our result is that it is diagonal; we only need to know the absolute square of $\widetilde{M}$, not its phase, in order to calculate $\operatorname{Im} M_{\mathrm{el}}$. We have an "optical theorem" at each impact parameter. The integral over impact parameter gives the ordinary (observable in practice) optical theorem. Unfortunately, however, impact parameter is not in practice accessible to experimental measurement. We need to know the phase in order to transform to transverse momentum.

The technique presented here can be used to investigate the high energy elastic scattering predicted by any model for multiparticle production. This method is especially useful as a qualitative tool. The calculation is done in a series of three steps. First, the multiparticle amplitude is transformed to impact parameter. An uncertainty principle can of ten be invoked at this step. The transverse momentum distribution, $\mathrm{do} / \mathrm{d} k$, is experimentally measured, and therefore constrains the distribution in the conjugate variable, which is impact parameter. The second step is to use beam depletion at each impact parameter, by summing over each cross section an using angular momentum conservation as expressed by $b=\Sigma x_{j} b_{j}$. At this step one does not need to worry about cancellations from different contributions; all cross sections are positive, so any contribution can only add to the amount of beam depletion. The third step is to transform the elastic amplitude to momentum space and compare with elastic scattering data. This third step is most conveniently done in reverse. The high energy elastic scattering amplitude is dominantly imaginary. Thus, the amplitude is essentially known and can be transformed to impact parameter. Moreover, the elastic scattering itself contributes a rather large, and known, amount to the unitarity sum building itself. This contribution can be subtracted, yielding the "overlap function" introduced by Van Hove [3]. This overlap function is the beam depletion caused by multiparticle scattering, and can be compared with the beam depletion of the model amplitude.

Such a comparison, in addition to testing existing models, can suggest new models whose structure naturally corresponds to the overlap function. An application of this technique to the multipheral model is the subject of another paper [4], and new models will be proposed in future publications.

In summary, a new way of carrying out unitarity calculations has been presented. This method involves 1) transforming from transverse momenta of produced particles to the conjugate variables, which are called "impact parameters", 2) using beam depletion and angular momentum conservation to calculate elastic scattering in impact parameter, and 3) transforming back to momentum transfer. Eqs. (1), (6) and (7) express these three steps. This method can be applied to multiparticle models, both existing and new.

\section{References}

[1] Similar expressions have been given by R.C. Hwa, Oregon preprint, January 1973 and by G. Calucci and R. Jengo, Lett. al Nuovo Cim. 5 (1972) 373.

[2] L. Caneschi and A. Schwimmer, Nucl. Phys. B44 (1972) 31.

[3] L. Van Hove, Nuovo Cim. 28 (1963) 798.

[4] F.S. Henyey, to be published. 